\title{
The secreted L-arabinose isomerase displays anti-hyperglycemic effects in mice
}

\author{
Moez Rhimi ${ }^{1,2^{*}}$, Luis G. Bermudez-Humaran ${ }^{3,4}$, Yuan Huang ${ }^{1,2}$, Samira Boudebbouze ${ }^{1,2}$, Nadia Gaci, ${ }^{1,2}$, \\ Alexandrine Garnier ${ }^{1,2}$, Jean-Jacques Gratadoux ${ }^{3,4}$, Héla Mkaouar ${ }^{1,2}$, Philippe Langella ${ }^{3,4}$ \\ and Emmanuelle Maguin ${ }^{1,2^{*}}$
}

\begin{abstract}
Background: The L-arabinose isomerase is an intracellular enzyme which converts L-arabinose into L-ribulose in living systems and D-galactose into D-tagatose in industrial processes and at industrial scales. D-tagatose is a natural ketohexose with potential uses in pharmaceutical and food industries. The D-galactose isomerization reaction is thermodynamically equilibrated, and leads to secondary subproducts at high $\mathrm{pH}$. Therefore, an attractive L-arabinose isomerase should be thermoactive and acidotolerant with high catalytic efficiency. While many reports focused on the set out of a low cost process for the industrial production of D-tagatose, these procedures remain costly. When compared to intracellular enzymes, the production of extracellular ones constitutes an interesting strategy to increase the suitability of the biocatalysts.
\end{abstract}

Results: The L-arabinose isomerase (L-Al) from Lactobacillus sakei was expressed in Lactococcus lactis in fusion with the signal peptide of usp45 (SP Usp45) $_{5}$. The L-Al protein and activity were detected only in the supernatant of the induced cultures of the recombinant L. lactis demonstrating the secretion in the medium of the intracellular L. sakei L-Al in an active form. Moreover, we showed an improvement in the enzyme secretion using either (1) L. lactis strains deficient for their two major proteases, ClpP and HtrA, or (2) an enhancer of protein secretion in L. lactis fused to the recombinant L-Al with the SP Usp45. Th L-Al enzyme secreted by the recombinant L. lactis strains or produced intracellularly in E. coli, showed the same functional properties than the native enzyme. Furthermore, when mice are fed with the L. lactis strain secreting the L-Al and galactose, tagatose was produced in vivo and reduced the glycemia index.

Conclusions: We report for the first time the secretion of the intracellular L-arabinose isomerase in the supernatant of food grade L. lactis cultures with hardly display other secreted proteins. The secreted L-Al originated from the food grade L. sakei $23 \mathrm{~K}$ was active and showed the same catalytic and structural properties as the intracellular enzyme. The L. lactis strains secreting the L-arabinose isomerase has the ability to produce D-tagatose in vivo and conferred an antihyperglycemic effect to mice.

Keywords: L-Arabinose isomerase, Secretion, Tagatose, Glycemia, Mice

\section{Background}

The $\mathrm{L}$-arabinose isomerase (L-AI, EC 5.3.1.4) is an enzyme that mediates the conversion of $\mathrm{L}$-arabinose to L-ribulose in vivo. At industrial scale this enzyme is used for the conversion of D-galactose into D-tagatose, thus

\footnotetext{
*Correspondence: moez.rhimi@jouy.inra.fr; emmanuelle.maguin@jouy. inra.fr

${ }^{1}$ INRA, UMR 1319 Micalis, Interactions of Firmicutes With Their

Environments, 78352 Jouy-en-Josas Cedex, France

Full list of author information is available at the end of the article
}

it is also referred to as a D-galactose isomerase [1]. The $\mathrm{D}$-tagatose is a D-fructose isomer currently used as a low calorie sweetener [2]. This ketohexose is a rare natural sugar tasting as sucrose and having similar physical properties [3] although it is not metabolized in humans and consequently has a very low caloric effect $[4,5]$. In addition to its sweetener properties, D-tagatose is an anti-hyperglycemic factor and exhibits an efficient antibiofilm effect. Later, the D-tagatose was considered as a 
GRAS "Generally Recognized as Safe" sweetener which can be used as a sugar substitute [6].

As previously reported, the isomerization of $\mathrm{D}$-galactose into D-tagatose is thermodynamically equilibrated allowing the shift of the reaction towards the tagatose production when the temperature is increased [6, 7]. Thus, several thermoactive L-AIs have been isolated from thermophilic microorganisms including Thermotoga, Bacillus and Thermus genera [8-10]. However when performed under alkaline conditions, the isomerization has several drawbacks mainly the production of undesirable sub-products [6, 11]. In order to improve the L-AIs suitability for biotechnological applications, many tools have been used such as: the screening of biodiversity to identify relevant enzymes with interesting properties, protein isolation, molecular modeling and rational design [12-14]. In this context, the thermoactivity, the metallic ions requirement and the catalytic efficiency of several enzymes were probed $[15,16]$. Recently, new production procedures of D-tagatose were described. We reported the concomitant bioconversion of D-galactose and D-glucose into D-tagatose and D-fructose, respectively. This original procedure was developed through the co-expression of a D-glucose isomerase and an L-arabinose isomerase in E. coli [17]. Another study [18] described the hydrolysis of lactose by the $\beta$-galactosidase from Pichia pastoris to galactose and glucose and the bioconversion of $30 \%$ of D-galactose into D-tagatose with the addition of the L-AI from Arthrobacter sp. While several studies revealed that numerous efforts have been made to set out a low cost procedure for the industrial production of tagatose, these processes remain costly [19]. This is mainly due to the biocatalyst production costs. Indeed, the L-AIs are intracellular enzymes that require not only the development of profitable procedures for their overexpression but also for their extraction and purification. Compared to the intracellular enzymes, the production of extracellular biocatalysts is an attractive alternative to improve the industrial process profitability.

Although the proteins belonging to the isomerase family are intracellular, in this study we report for the first time an efficient secretion in the extracellular medium of the L-AI from Lactobacillus sakei $23 \mathrm{~K}$ by the food grade bacterium Lactococcus lactis. We investigated the properties of the secreted protein and its efficacy to bioconvert galactose into tagatose. We also investigated the functionality of the enzyme, the in vivo isomerization of galactose and the cognate anti-hyperglycemic effect of the produced tagatose in mice model.

\section{Results and discussion}

\section{L-Al secretion in L. lactis clpP-htrA strain}

$\mathrm{L}$-AI is an intracellular protein, which is an efficient bioconverter of galactose into tagatose. This latter property confers to this enzyme family a strong industrial interest. However, the tagatose production remains to be improved mainly in terms of efficiency and process cost. Many efforts have been focused on the improvement of the biochemical properties of L-AI enzymes and their adaptation to industrial processes. However although the extraction and purification of the intracellular L-AIs increase the cost of production of these biocatalysts, the process steps have hardly been addressed. In this context, we investigated whether an intracellular L-AI could be produced in the extracellular medium. We choose L. lactis as the heterologous host for the expression and secretion test because (1) it is a food grade bacterium, (2) it secretes only one detectable extracellular protein (Usp45) [20], (3) it possesses only two major proteases, namely ClpP (intracellular) and HtrA (extracellular) (4) it has been used for the successful expression and secretion of several proteins of medical and industrial interest and for in situ delivery [21].

The gene encoding the L-AI from L. sakei $23 \mathrm{~K}$ was cloned under the control of $\mathrm{p}_{n i s}$, the nisin-inducible promoter, either directly or in fusion with the signal peptide originated from the L. lactis usp 45 gene. Both constructions as well as the empty vector were transformed in L. lactis NZ9000. The activity test showed that the L-AI activity is only found in the Nisin-induced cultures of recombinant $L$. lactis NZ9000 strain and its clpP-htrA derivative. No activity was observed in the strains harboring the empty plasmid. As expected, L-AI activity was clearly detected $(21 \pm 0.2 \mathrm{U} / \mathrm{mg})$ in the cell fraction of L. lactis NZ9000 strain harboring the PCYT:araA plasmid, whereas no signal was detected in the supernatant (Table 1). Similar analysis of L. lactis NZ9000 strain harboring the pSEC:araA plasmid resulted in an L-AI activity of $(10 \pm 0.3 \mathrm{U} / \mathrm{mg})$ in the supernatant fraction while not activity was detected in the cell fraction (Table 1). No activity was found in the cellular extracts from the cultures corresponding to the strains harbouring empty vectors. These results demonstrated for the first time that the L. sakei $23 \mathrm{~K} \mathrm{~L}-\mathrm{AI}$ is expressed in an active form in L. lactis not only in its intracellular form but also as a secreted enzyme.

SDS-PAGE analysis of secreted L-AI revealed the presence of a protein band with an apparent molecular weight of about $56 \mathrm{kDa}$ (Fig. 1a). Mass spectrometry experiments confirmed the identity of this band as the L-AI from L. sakei $23 \mathrm{~K}$ (data not shown). The ability to express the L-AI from L. sakei in L. lactis is in agreement with a recent report describing the expression of the Bifdobacterium longum L-AI in L. lactis [18]. However to the best of our knowledge, there is so far no report on the secretion of the L-AI. This work constitutes not only the first report on the secretion of an L-AI, but also an 
Table 1 L-Al specific activity determination in L. lactis NZ9000 harboring the secreted and intracellular enzyme forms

\begin{tabular}{lll}
\hline Construction & \multicolumn{2}{l}{ Specific activities (U/mg) } \\
\cline { 2 - 3 } & Supernatant & $\begin{array}{l}\text { Protein crude } \\
\text { extract }\end{array}$ \\
\hline NZ9000/pCYT:araA & ND & $21 \pm 0.2$ \\
NZ9000/pSEC:araA & $10 \pm 0.3$ & ND \\
NZ9000/pSEC:LEISS:araA & $13 \pm 0.8$ & ND \\
\hline
\end{tabular}

ND not detected

attractive way to produce this protein for biotechnological applications.

To increase the L-AI secretion by L. lactis we used the pSEC:LEISS plasmid [22] which contains the usp 45 signal peptide fused to 15 nucleotides that encodes to a 9-residue synthetic propeptide, LEISSTCDA acting as an enhancer of protein secretion in L. lactis (Table 2). The L-AI activity was monitored in the supernatants of the L. lactis NZ9000 strains harboring the pSEC:araA and pSEC:LEISS:araA plasmids. The L-AI activities measured in the culture supernatants were $10 \pm 0.3$ and $13 \pm 0.8$ $\mathrm{U} / \mathrm{mg}$ for the strains carrying the pSEC:araA and the pSEC:LEISS:araA, respectively i.e. about half of the activity measured in the crude extract of NZ9000 carrying the pCYT:araA vector allowing the intracellular accumulation of the enzyme.

It is well known that heterologous proteins can be targeted by the proteases of the expressing host. We investigated the effect of the L. lactis proteases on the yield of L-AI production and secretion. Three isogenic mutants of the NZ9000 strain were used; they are deficient in each one or in both proteases of L. lactis; the intracellular protease $\mathrm{ClpP}$ and/or the extracellular cell surface protease HtrA (Table 2). As shown in Table 3, the measured activity for the $c l p P$ mutant, the $h t r A$ mutant and the $c l p P$ htr $A$ double mutant were $17 \pm 0.4,19 \pm 0.3$ and $22 \pm 0.6$ $\mathrm{U} / \mathrm{mg}$ compared to $13 \pm 0.8 \mathrm{U} / \mathrm{mg}$ obtained in the wildtype NZ9000 strain carrying the same plasmid. These data indicate that each of the $L$. lactis proteases affects the yield of the secreted L-AI.

Altogether, the best production levels of the L-AI in $L$. lactis were obtained (1) in the pellet using the pCYT:araA $(21 \pm 0.2 \mathrm{U} / \mathrm{mg})$ and (2) directly in the supernatant with the combination of the L. lactis clpP-htrA deficient strain with the pSEC:LEISS:araA expression plasmid $(22 \pm 0.3 \mathrm{U} / \mathrm{mg})$.

\section{Functional characterization of the secreted L-AI}

To study the biochemical properties of the secreted and the intracellular $\mathrm{L}$-AI we purified this protein from $L$.

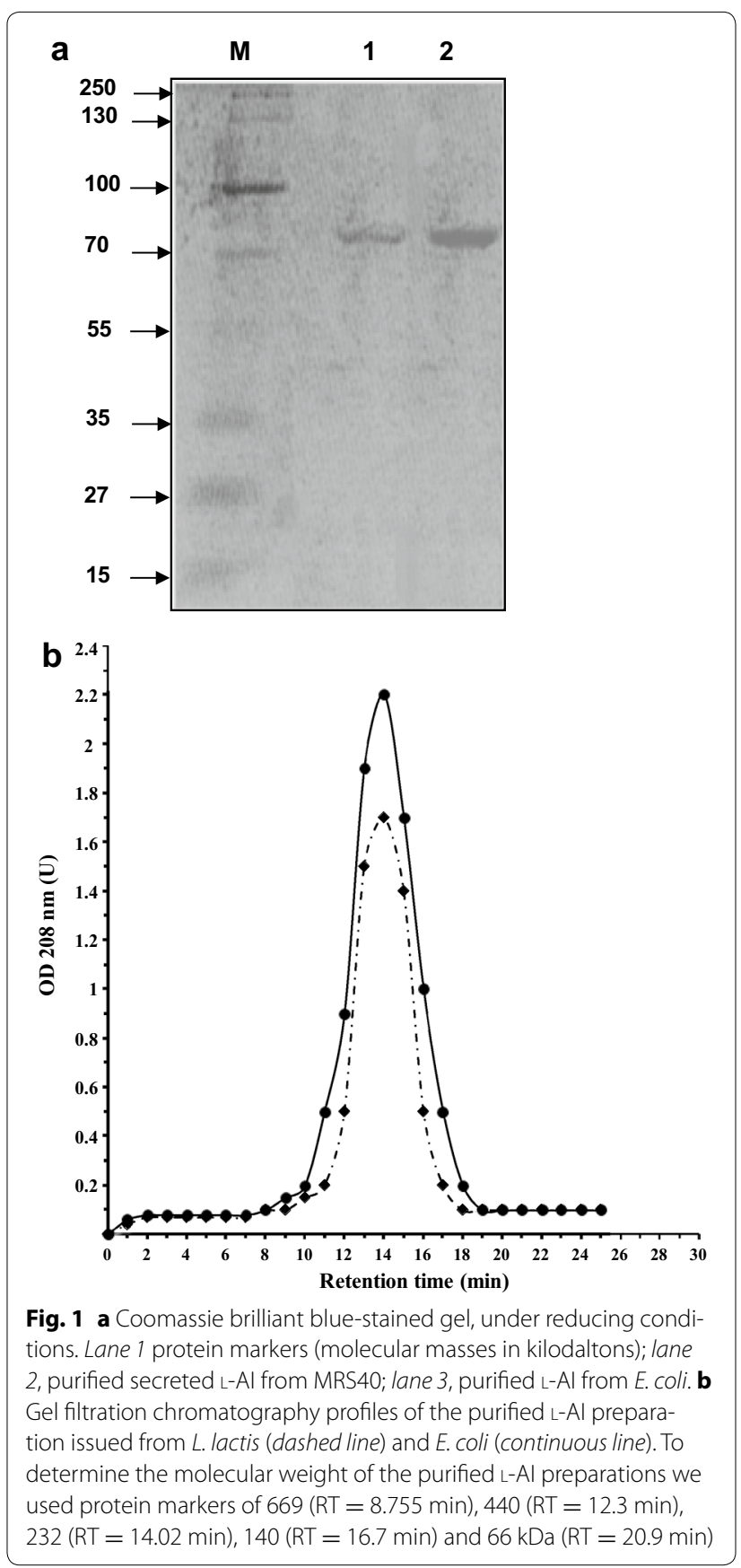

lactis and E. coli, respectively. The analysis of the purified protein from E. coli and L. lactis by SDS-PAGE and gel filtration chromatography showed that both purified enzymes have a tetrameric arrangement (Fig. 1). These results evidenced that the secreted L-AI monomer has a functional tetrameric arrangement in the culture supernatant. This observation is also supported by the fact that the secreted $L$. sakei $\mathrm{L}$-AI and that all L-AIs reported so far, are active as homotetramers or homohexamers [7, 23]. 
Table 2 Bacterial strains and plasmids used in this work

\begin{tabular}{|c|c|c|}
\hline Strains & Genotypes & References \\
\hline MRS36 & Escherichia coli BL21 strain harboring the pMR36 plasmid & [30] \\
\hline NZ9000 & MG1363 (nisRK genes in chromosome), plasmid free & {$[33]$} \\
\hline NZ9000 htrA & NZ9000 defective for the htrA gene, plasmid free & {$[29]$} \\
\hline NZ9000 clpP & NZ9000 defective for the clpP gene, plasmid free & [34] \\
\hline NZ9000 htrA clpP & NZ9000 defective for the htrA and the clpP genes, plasmid free & {$[35]$} \\
\hline MRS37 & NZ9000 harbouring the psec:LEISS:araA & This work \\
\hline MRS38 & NZ9000 clpP harbouring the psec:LEISS:araA & This work \\
\hline MRS39 & NZ9000 htrA harbouring the psec:LEISS:araA & This work \\
\hline MRS40 & NZ9000 htrAclpP harbouring the psec:LEISS:araA & This work \\
\hline Plasmid & Construct & References \\
\hline pMR36 & Plasmid encoding the L-arabinose isomerase encoding gene (araA) from L. sakei $23 \mathrm{~K}$ & {$[30]$} \\
\hline $\mathrm{pCYT}$ & Vector derived from pGK12 carrying a chloramphenicol resistance gene and the $\mathrm{p}_{\text {nis }}$ inducible promoter & {$[22]$} \\
\hline pSEC & $\begin{array}{l}\text { Vector derived from pGK12 carrying a chloramphenicol resistance gene and the } \mathrm{p}_{\text {nis }} \text { inducible promoter fused to the } \\
\text { signal peptide of the usp } 45 \text { gene }\end{array}$ & {$[22]$} \\
\hline pSEC:LEISS & $\begin{array}{l}\text { Vector derived from pGK12 carrying a chloramphenicol resistance gene and the } p_{\text {nis }} \text { inducible promoter in front of the } \\
\text { signal peptide of usp45 fused to a sequence encoding the LEISTCDA polypeptide }\end{array}$ & {$[22]$} \\
\hline pCYT:araA & pCyt carrying the L. sakei araA gene under the control of the $\mathrm{p}_{\text {nis }}$ promoter & This work \\
\hline pSEC:araA & pSec carrying the L. sakei araA gene fused to the signal peptide of usp45and under the control of the $p_{\text {nis }}$ promoter & This work \\
\hline pSEC:LEISS:araA & $\begin{array}{l}\text { pSec:LEISS carrying under the control of the } \mathrm{p}_{\text {nis }} \text { promoter, the signal peptide of usp45 associated with the LEISSTCDA } \\
\text { encoding fragment and fused to the L. sakei araA gene }\end{array}$ & This work \\
\hline
\end{tabular}

Table 3 Effect of the L. lactis mutant strains on the enzyme secretion efficiency

\begin{tabular}{lll}
\hline Strain & Strain designation & Specific activity $\mathbf{( U / \mathbf { m g } )}$ \\
\hline NZ9000 & MRS37 & $13 \pm 0.8$ \\
NZ9000 clpP & MRS38 & $17 \pm 0.4$ \\
NZ9000 htrA & MRS39 & $19 \pm 0.3$ \\
NZ9000 htrAclpP & MRS40 & $22 \pm 0.6$ \\
\hline
\end{tabular}

To go one step further, we analyzed both purified protein fractions by circular dichroism. Our results revealed that the purified proteins from L. lactis (secreted form) and $E$. coli (intracellular form) exhibited similar conformations (Fig. 2).

In addition, we studied the activity of the two purified protein preparations as function of the temperature and the $\mathrm{pH}$. Both proteins fractions displayed the same optimal temperature $\left(30-40{ }^{\circ} \mathrm{C}\right.$ ) and optimal $\mathrm{pH}$ (5.0-7.0). The kinetic studies demonstrated that the purified LAI from $L$. lactis and E. coli had a catalytic efficiency of $65 \pm 0.8$ and $64 \pm 0.2 / \mathrm{mM} / \mathrm{min}$ for L-arabinose, respectively. These results established that the secreted protein not only have the same tetrameric arrangement as the protein over-expressed and purified from E. coli, but also the same biochemical and kinetic properties. This underlines again the efficiency of the secretion of this L-AI in L. lactis and the relevance of this mode of production for

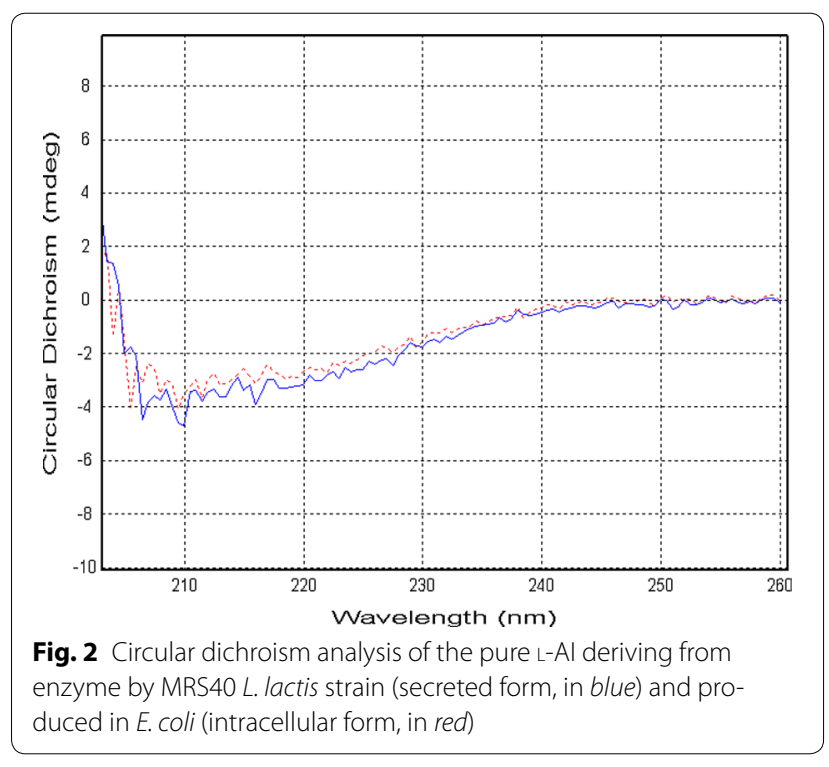

industrial applications. Such procedure constitutes an original promising tool for the secretion of this protein family in this food-grade host microorganism.

\section{The secreted enzyme efficiently bioconverts D-galactose into D-tagatose}

The ability of the MRS40 strain to bioconvert the D-galactose into D-tagatose was studied. The induced cells are 
able to produce the $\mathrm{D}$-tagatose from the $\mathrm{D}$-galactose with a production rate of $32 \%$ at $\mathrm{pH} 5.0,6.0$ and 7.0 (Fig. 3). In contrast in the same conditions, the non-induced MRS40 cells did not produce detectable amount of D-tagatose and the galactose concentration remained stable (data not shown). The purified secreted L-AI displayed a high efficiency to isomerize the D-galactose into D-tagatose (Fig. 3). The analysis of the kinetic of D-galactose isomerization revealed that the bioconversion rates were higher with the purified enzyme (Fig. 3) than with the nisininduced MRS40 strain. In addition, the highest bioconversion rate was achieved after $5 \mathrm{~h}$ in case of the purified enzyme and $7 \mathrm{~h}$ with the induced MRS40 cells (Fig. 3). It is likely to be related to a higher concentration of the purified enzyme compared to the amount of L-AI enzyme directly secreted by the induced bacterial culture.

As shown in Fig. 3, the conversion rates were not significantly altered when the $\mathrm{pH}$ varied both in case of the induced MRS40 culture and of the purified enzyme. Such phenomenon may be explained by the wide range activity of the L. sakei L-AI [24].

These results highlighted the ability of the induced MRS40 cells and of the purified secreted L-AI to biocatalyze the $\mathrm{D}$-tagatose production even at neutral and low $\mathrm{pH}$. This underlines again the efficiency of the L-AI secretion as a powerful system for the over-production of an active extracellular L-AI enzyme.

\section{The produced D-tagatose in vivo has an antihyperglycemic} effect in mice

The use of tagatose in pharmaceutical applications has been previously reported. One of the main properties

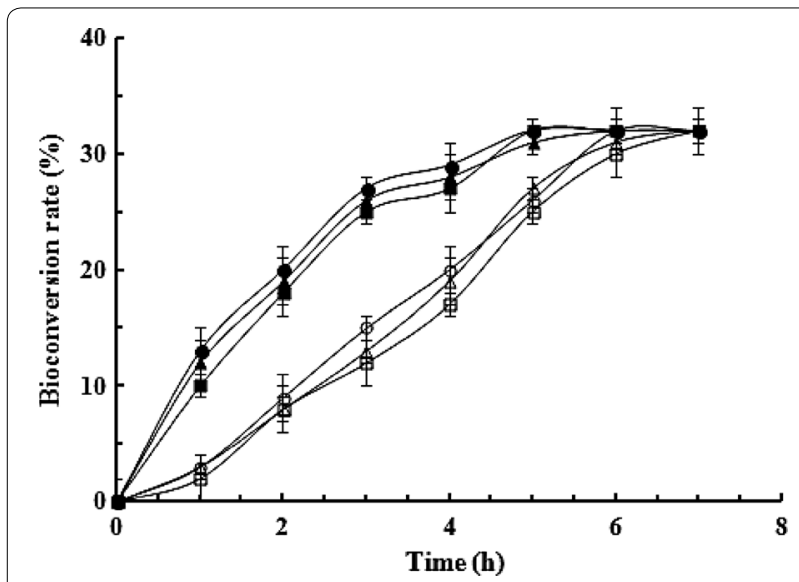

Fig. 3 Bioconversion of the D-galactose into D-tagatose at different $\mathrm{pH}$. The conversion was performed by either (1) the induced MRS40 strain: pH 5 (open circle), pH 6 (open triangle), pH 7 (open square) or (2) the purified secreted L-Al enzyme pH 5 (filled circle), pH 6 (filled triangle), $\mathrm{pH} 7$ (filled square) of this natural sugar is its anti-hyperglycemic effect [4]. To investigate this property, we studied the impact of the L. lactis strain secreting the L-AI and of the purified secreted enzyme on the glycemia of mice receiving D-galactose. This experimental procedure will also evidence the in vivo activity of the L-AI and the ability of the L. lactis strain to actively synthesize the L-AI in vivo. As illustrated in Fig. 4, the treatment of mice with D-galactose increased the glycemia values induced by glucose administration when compared to the results obtained with PBS only. On contrast, when the D-glucose administration was preceded by D-tagatose ingestion, the glycemia values did not increased (Fig. 4). These data highlighted the anti-hyperglycemic role of $\mathrm{D}$-tagatose and confirmed what was previously reported regarding the glycemia protecting effect of this natural sugar $[4,25$, 26]. We then investigated the efficiency of the secreted $\mathrm{L}$-AI to produce the D-tagatose in vivo and to protect mice against a glycemia increase. As shown in Fig. 4, both non induced MRS40 and L. lactis clpP htrA/pSec:LEISS strain did not decreased the mice glycemia values after the administration of glucose. Moreover, the analysis of these glycemia profiles revealed that they were similar to that of the PBS control. The pretreatment of the mice with the nisin-induced MRS40 cells and D-galactose allowed a certain level of protection against the glycemia increase in comparison to the mice treated with PBS and D-galactose. These data strongly suggests that

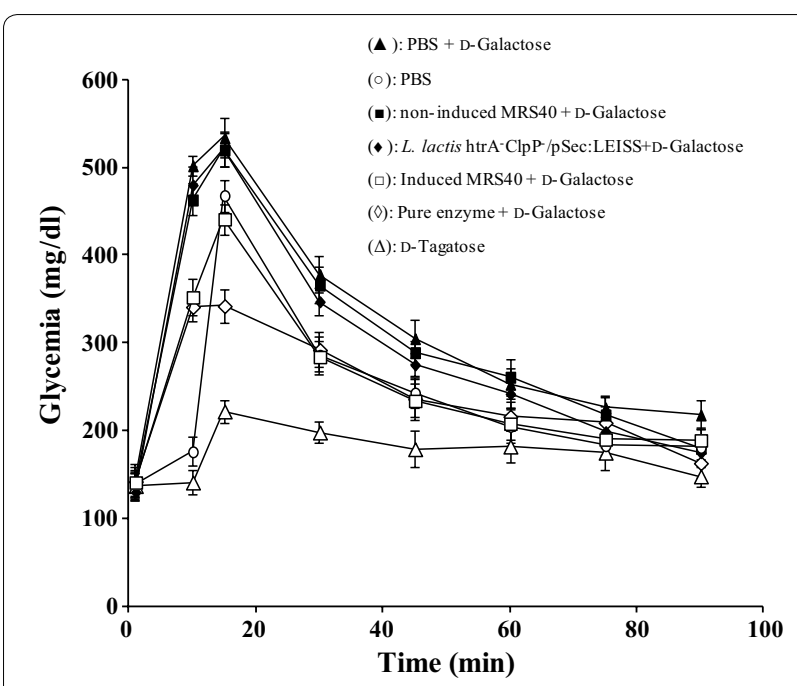

Fig. 4 Effect of tagatose on the glycemia in mice. Tests were carried out by administration of tagatose solution or through bioconversion of galactose in vivo using the pure secreted enzyme and the induced MRS40 strain. Filled triangle PBS + D-galactose, filled square noninduced MRS40, filled diamond L. lactis htrA- $\mathrm{ClpP}^{-} / \mathrm{pSec}$ :LEISS, open circle PBS, open diamond pure enzyme +D-galactose, open square induced MRS40, open triangle D-tagatose 
the induced MRS40 strain secreted an active L-AI which was able to bioconvert in vivo the administered galactose into tagatose leading to the glycemia protection typical of this natural sugar. This interpretation is strongly supported by the fact that the mice treated with the purified L-AI enzyme and the D-galactose also showed a significant protection against the induced hyperglycemia (Fig. 4). The decrease of hyperglycemia was the highest after D-tagatose ingestion, intermediate with the ingestion of the purified enzyme and galactose and lower with the ingestion of the induced MRS40 strain and galactose. This observation is in agreement with (1) the elevated amount of D-tagatose used in this experiment $(2 \mathrm{mg} /$ mice likely to be in excess), (2) the higher bioconversion activity reached with the purified enzyme compared to that obtained with the induced MRS40 culture as established in our in vitro experiments (Fig. 3). Altogether our results revealed for the first time that the purified and secreted L-AIs convert in vivo the galactose into tagatose as shown by its protective effect against the hyperglycemia observed in the animal model.

\section{Conclusions}

Here we report the secretion of the first L-AI from $L$. sakei $23 \mathrm{~K}$, belonging to the isomerase family, in the food-grade L. lactis microorganism. The secreted L-AI from $L$. sakei $23 \mathrm{~K}$ displays the same biochemical and structural features when compared to the L-AI produced intracellularly in E. coli. Moreover, the L. lactis secreted L-AI efficiently bioconverted the D-galactose into D-tagatose in vivo displaying thus an anti-hyperglycemic effect in mice. In the near future we will focus our efforts on the study of the effect of tagatose concentration on the anti-hyperglycemic response. Furthermore, the optimization and/or isolation of new L-AI having high catalytic efficiency and stability will be of interest for the tagatose production at industrial scale. Such features stress the efficiency of the secretion of L-AI as a profitable way to produce active L-AI for industrial applications.

\section{Methods}

Bacterial strains, media, plasmids and growth conditions

The bacterial strains and plasmids used in this work are listed in Table 2. E. coli was grown in Luria-Bertani medium at $37{ }^{\circ} \mathrm{C}$ [27]. L. lactis was grown in M17 medium supplemented with $1 \%$ glucose at $30{ }^{\circ} \mathrm{C}$ without agitation. Plasmids were transformed in L. lactis by electroporation [28]. Plasmids were selected by using antibiotics: $5 \mu \mathrm{g}$ of chloramphenicol per $\mathrm{ml}$ for $L$. lactis, $10 \mu \mathrm{g}$ of chloramphenicol per $\mathrm{ml}$ for $E$. coli, and $100 \mu \mathrm{g}$ of ampicillin per $\mathrm{ml}$ for $E$. coli. Nisin was used at final concentration of $10 \mathrm{ng} / \mathrm{ml}$ for L. lactis. $1 \mathrm{mM}$ of IPTG was used with E. coli.

\section{PCR and DNA manipulation}

Preparation of plasmid DNA was performed using the Miniprep kit (Promega). DNA digestion with restriction endonucleases and separation of fragments in agarose gel electrophoresis were performed as described by Sambrook et al. [27]. Polymerase chain reactions were carried out in a Gene Amp PCR System 9700 (Applied Biosystems). The amplification reaction mixtures $(100 \mu \mathrm{l})$ contained Phusion High-Fidelity DNA polymerase buffer, $10 \mathrm{pmol}$ of each primer, $50 \mathrm{ng}$ of DNA template, and 10 units of High-Fidelity DNA polymerase (Fermentas). The cycling parameters were $94{ }^{\circ} \mathrm{C}$ for 5 min, followed by 35 cycles at $94{ }^{\circ} \mathrm{C}$ for $30 \mathrm{~s}, 55{ }^{\circ} \mathrm{C}$ for $30 \mathrm{~s}$ and $72{ }^{\circ} \mathrm{C}$ for $90 \mathrm{~s}$ and finally $7 \mathrm{~min}$ at $72{ }^{\circ} \mathrm{C}$. PCR products were purified using the $\mathrm{GFX}^{\mathrm{TM}}$ PCR DNA and Gel Band Purification Kit (Amersham Bioscience), following the manufacturer's instructions.

\section{L-Arabinose isomerase molecular cloning strategy}

The pMR36 plasmid was used as a template for the amplification of the gene encoding the L-AI from Lactobacillus sakei $23 \mathrm{~K}$ (Table 2). Two primers F-araA: AACTG CAGCATTAAATACAGAAAATTATGAATTTTGG and R-araA: GGACTAGTCCTTATTTAATATTGACGTAAG TCAAATC were used to amplify the araA gene flanked by the SpeI and PstI restriction sites. The resulting PCR fragment was purified and then digested with the latter restriction enzymes. The digested fragment was purified and subsequently ligated to the pCYT, pSEC and pSEC:LEISS vectors (to obtain pCYT:araA, pSEC:araA and pSEC:LEISS:araA, respectively) linearized with SpeI and NsiI restriction enzymes (Table 2). The ligation products were then transformed into either L. lactis NZ9000 or L. lactis clpP-htrA strain [29]. Recombinant clones were analyzed by restriction and generated constructions were confirmed by DNA sequencing using an automated DNA sequencer (MWG Eurofins).

\section{Preparation of crude extracts and protein purification}

Recombinant $L$. lactis were grown until $\mathrm{OD}_{600}=0.6$ and induction with $10 \mathrm{ng} / \mathrm{ml}$ of nisin (Sigma) was performed during $3 \mathrm{~h}$. The cells were harvested and the supernatant was concentrated using a $100 \mathrm{kDa}$ cut-off membrane. The resulting protein fraction was subjected to an anion exchange chromatography (Mono-Q 5/50GL, GE Healthcare). Purification was achieved by a size exclusion chromatography step (S200 column, Amersham Bioscience) using an ÄKTA purifier system (Amersham Biosciences). The used buffer was $100 \mathrm{mM}$ sodium acetate (pH 5.0) and elution fractions were $0.5 \mathrm{ml}$.

The harvested L. lactis cells were washed twice with $100 \mathrm{mM}$ sodium acetate buffer ( $\mathrm{pH}$ 5.0) and disrupted by glass beads (diameter of 212-300 $\mu \mathrm{m}, \mathrm{v} / \mathrm{v}$, Sigma). Crude 
cell extract were recovered by centrifugation $(30,000 \times g$, $20 \min$ at $4{ }^{\circ} \mathrm{C}$ ).

Lactobacillus sakei L-AI produced in E. coli strain (MRS36) was over-expressed and purified as previously reported [30].

\section{Protein quantification and electrophoresis}

Protein concentrations were determined using the Bradford method with bovine serum albumin as standard. The purified enzyme samples were migrated in $12 \%$ sodium dodecyl sulfate-polyacrylamide gel electrophoresis (SDS-PAGE) according to the method of Laemmli [31]. Protein bands were visualized by Coomassie brilliant blue R-250 (BioRad) staining.

\section{Enzyme assays}

L-AI activity was established by determining the amount of generated L-ribulose or D-tagatose. Under standard conditions, the reaction mixture contained $0.8 \mathrm{mM} \mathrm{Mg}^{2+}, 0.8 \mathrm{mM} \mathrm{Mn}^{2+}, 50 \mu \mathrm{l}$ of enzyme preparation at a suitable dilution; $5 \mathrm{mM}$ of $\mathrm{L}$-arabinose (or D-galactose) and sodium acetate buffer $100 \mathrm{mM}$ (pH 5.0) to bring the final volume to $1 \mathrm{ml}$. The reaction mixture was incubated at $35^{\circ} \mathrm{C}$ during 1 or $10 \mathrm{~min}$ for L-arabinose and $\mathrm{D}$-galactose, respectively, followed by the incubation of the samples at $99^{\circ} \mathrm{C}$ during $5 \mathrm{~min}$. The generated L-ribulose (or D-tagatose) was measured by the cysteine carbazole sulfuric-acid method, and the absorbance was measured at $560 \mathrm{~nm}$ [32]. The D-tagatose production was also confirmed by high-pressure liquid chromatography (HPLC).

One unit of L-AI activity was defined as the amount of enzyme catalyzing the formation of $1 \mu \mathrm{mol}$ keto-sugar per min under the above-specified conditions.

\section{Circular dichroism experiments}

Circular dichroism (CD) measurements were done with chirascan spectropolarimeter (Applied photophysics). Purified proteins were used at concentration of $1 \mathrm{mg} / \mathrm{ml}$. The CD spectra of enzyme samples in a cuvette $(0.1 \mathrm{~cm})$ path length were analyzed in the far-UV region comprised between 200 and $280 \mathrm{~nm}$. Scans were collected at $0.1 \mathrm{~nm}$ intervals with a $1 \mathrm{~nm}$ bandwidth five times. Each spectrum was corrected by subtracting that of the solution containing the used buffer.

\section{Biochemical and kinetic characterization}

The effect of temperature on the activity was determined by incubating the purified enzyme at temperatures ranging from 4 to $55^{\circ} \mathrm{C}$, whereas the $\mathrm{pH}$ profile was obtained by measuring the activity at various $\mathrm{pH}$ values from 3.0 to 8.5 [3.0-5.0 with sodium acetate buffer, $6.0-7.0$ with 2-morpholinoethanesulfonic acid (MES) buffer and 7.58.5 with Bicine buffer].

Kinetic properties were studied on the basis of Lineweaver-Burk plots. Assays were done in $100 \mathrm{mM}$ sodium acetate buffer ( $\mathrm{pH} 5.0$ ), $0.8 \mathrm{mM} \mathrm{Mg}{ }^{2+}, 0.8 \mathrm{mM}$ $\mathrm{Mn}^{2+}$ and 1-800 $\mathrm{mM}$ substrate (L-arabinose or D-galactose). Samples were incubated at $35{ }^{\circ} \mathrm{C}$ and the amount of keto-sugar generated (L-ribulose or D-tagatose) was determined by the cysteine-carbazole-sulfuric acid method [32].

\section{In vitro D-galactose bioconversion}

Induced MRS40 cells and purified secreted L-AI with a final concentration of $10^{9} \mathrm{CFU} / \mathrm{ml}$ and $0.3 \mathrm{mg} / \mathrm{ml}$, respectively, were used to produce $\mathrm{D}$-tagatose. Reactions were carried out under different $\mathrm{pH}$ values $(\mathrm{pH}$ 5.0, 6.0 and 7.0) by using $10 \mathrm{~g} / \mathrm{l} \mathrm{D}$-galactose. D-tagatose was determined by cysteine carbazole method [32].

\section{Animal experiments and glycemia measurement}

Male C57BL/6 mice (6-8 weeks old) (Janvier, Le Genest 428 Saint Isle, France or Taconic mice New York, USA) were maintained at the animal care facilities of the National Institute of Agricultural Research (IERP, INRA, Jouy-en-Josas, France) under specific pathogen-free conditions. Mice were housed under standard conditions for a minimum of 1 week before experimentation. All experiments were performed in accordance with European Community rules and approved by the Animal Care Committee COMETHEA (Comité d'Ethique en Expérimentation Animale du Centre INRA de Jouy-en-Josas et AgroParisTech, Jouy en Josas, France). Food intake was stopped $6 \mathrm{~h}$ before starting experiments.

L. lactis cellular pellets were harvested by centrifugation $\left(3000 g\right.$, at $\left.4{ }^{\circ} \mathrm{C}\right)$ and washed three times with sterile PBS. The pellet was suspended in PBS to a final concentration of $10^{9} \mathrm{CFU}$. Groups of mice $(n=10)$ received a intragastric administration of either PBS, D-galactose $(10 \mathrm{~g} / \mathrm{l})$, D-tagatose $(10 \mathrm{~g} / \mathrm{l})$ or D-galactose $(10 \mathrm{~g} / \mathrm{l})$ with: (1) MRS40 strain carrying the pSEC:LEISS vector, (2) induced MRS40 strain carrying the pSEC:LEISS:araA vector, (3) non-induced MRS40 strain carrying the pSEC:LEISS:araA vector and (4) the purified enzyme $(0.3 \mathrm{mg} / \mathrm{ml})$. The administered volume was $0.2 \mathrm{ml}$ for each condition followed by a glucose challenge $(1 \mathrm{~g} / \mathrm{kg}$ body weight). Glycemia measurements were done by using the Accu-Chek performa system (Roche).

\section{Statistical analysis}

The data reported in this work were plotted using Sigma Plot (Version 9.0). Each value represents the mean for three independent experiments performed in duplicate. 


\section{Authors' contributions}

MR participated in the design of the study, experiments and writing the manuscript. LB contributed to the molecular biology assays, animal experiments and writing the manuscript. YH performed molecular biology experiments and enzyme assays. SB participated in the molecular biology and biochemical experiments. NG participates in the animal experiments. AG contributed to the biochemical and the molecular biology experiments. JG performed animal experiments. HM contributed to animal experiments. PL participated in the design of the work and helped in drafting the manuscript. EM contributed in the design, results analysis and writing the manuscript. All authors read and approved the final manuscript.

\begin{abstract}
Author details
${ }^{1}$ INRA, UMR 1319 Micalis, Interactions of Firmicutes With Their Environments, 78352 Jouy-en-Josas Cedex, France. ${ }^{2}$ AgroParisTech, Micalis, Interactions of Firmicutes With Their Environments, 78352 Jouy-en-Josas Cedex, France. ${ }^{3}$ INRA, UMR 1319 Micalis, Commensal and Probiotics-Host Interactions Laboratory, 78352 Jouy-en-Josas Cedex, France. ${ }^{4}$ AgroParisTech, Micalis, Commensal and Probiotics-Host Interactions Laboratory, 78352 Jouy-en-Josas Cedex, France.
\end{abstract}

\section{Acknowledgements}

The French National Institute for Agricultural Research (INRA) supported this work. We thanks Dr Michel JUY and Dr Michel BECCHI from "Institut de Biologie et Chimie des Protéines, Lyon" for their appreciated help in the biophysical experiments and mass spectrometry analysis, respectively.

\section{Competing interests}

The authors declare that they have no competing interests.

Received: 25 August 2015 Accepted: 27 November 2015

Published online: 21 December 2015

\section{References}

1. Cheetham PSJ, Woottom AN. Bioconversion of D-galactose into D-tagatose. Enzy Microb Technol. 1993;15:105-8.

2. Levin GV, Zehner LR, Saunders JP, Beadle JR. Sugar substitutes: their energy values, bulk characteristics, and potential health benefits. Am J Clin Nutr. 1995;62(Suppl 5):1161S-8S.

3. Mazur AW. Functional sugar substitutes with reduced calories. 1989. US Patent $N^{\circ} 5064672 \mathrm{~A}$.

4. Zehner LR, Levin G, Saunder JP, Beadle JR. D-tagatose as anti-hyperglycemiant agent. 1994. US patent 5,356,879.

5. Livesey G, Brown JC. D-tagatose is a bulk sweetener with zero energy determined in rats. J Nutr. 1996;126:1601-9.

6. Lee $\mathrm{D}$, Jang H, Choe EB, Lee $\mathrm{S}, \mathrm{Kim} \mathrm{S}$, Hong Y, et al. Characterization of a thermostable L-arabinose ( $\mathrm{D}$-galactose) isomerase from the hyperthermophilic eubacterium Thermotoga maritime. Appl Environ Microbiol. 2004;70:1397-404.

7. Rhimi M, Bejar S. Cloning, purification and biochemical characterization of metallic-ions independent and thermoactive L-arabinose isomerase from the Bacillus stearothermophilus US100 strain. Biochim Biophys Acta. 2006;1760:191-9.

8. Roh HJ, Kim P, Park YC, Choi JH. Bioconversion of D-galactose into D-tagatose by expression of L-arabinose isomerase. Biotechnol Appl Biochem. 2000;31:1-4.

9. Kim JW, Kim YW, Roh HJ, Kim HY, Cha JH, Park KH, et al. Production of tagatose by a recombinant thermostable L-arabinose isomerase from Thermus sp. IM6501. Biotechnol Lett. 2003;25:963-7.

10. Hong YH, Lee DW, Lee SJ, Choe EA, Kim SB, Lee YH, et al. Production of D-tagatose at high temperatures using immobilized Escherichia coli cells expressing L-arabinose isomerase from Thermotoga neapolitana. Biotechnol Lett. 2007;29:569-74.

11. Lee SJ, Lee DW, Choe EA, Hong YH, Kim SB, Kim BC, et al. Characterization of a thermoacidophilic L-arabinose isomerase from Alicyclobacillus acidocaldarius: role of Lys-269 in pH optimum. Appl Environ Microbiol. 2005;71:7888-96.

12. Kim P, Yoon SH, Seo MJ, Oh DK, Choi JH. Improvement of tagatose conversion rate by genetic evolution of thermostable galactose isomerase. Biotechnol Appl Biochem. 2001;34:99-102.
13. Prabhu P, Jeya M, Lee JK. Probing the molecular determinant for the catalytic efficiency of L-arabinose isomerase from Bacillus licheniformis Appl Environ Microbiol. 2010;76:1653-60.

14. Patel DH, Cho EJ, Kim HM, Choi S, Bae HJ. Engineering of the catalytic site of xylose isomerase to enhance bioconversion of a non-preferential substrate. Prot Eng Design Select. 2012;25:331-6.

15. Sang-Jae L, Sang JL, Yong-Jik L, Seong-Bo K, Sung-Kun K. Homologous alkalophilic and acidophilic L-arabinose isomerases reveal region-specific contributions to the $\mathrm{pH}$ dependence of activity and stability. Appl Environ Microbiol. 2012;78:8813-6.

16. Zhou X, Wu J. Heterologous expression and characterization of Bacillus coagulans L-arabinose isomerase. World J Microb Biotechnol. 2012;28:2205-12.

17. Rhimi M, Messaoud EB, Borgi MA, Ben Khadra K, Bejar S. Co-expression of L-arabinose isomerase and D-glucose isomerase in E. coli and development of an efficient process producing simultaneously D-tagatose and D-fructose. Enz Microb Technol. 2007;40:1531-7.

18. Wanarska M, Kur J. A method for the production of D-tagatose using a recombinant Pichia pastoris strain secreting $B$-D-galactosidase from Arthrobacter chlorophenolicus and a recombinant L-arabinose isomerase from Arthrobacter sp CC2. Microb Cell Fact. 2012;11:113.

19. Oh D. Tagatose: properties, applications, and biotechnological processes. Appl Microbiol Biotechnol. 2007;76:1-8.

20. Van Asseldonk M, Rutten G, Oteman M, Siezen RJ, de Vos WM, Simons G. Cloning of usp45, a gene encoding a secreted protein from Lactococcus lactis subsp. lactis MG1363. Gene. 1990;95:155-60.

21. Bermúdez-Humarán LG, Aubry C, Motta JP, Deraison C, Steidler L, Vergnolle $\mathrm{N}$, et al. Engineering lactococci and lactobacilli for human health. Curr Opin Microbiol. 2013;16:278-83.

22. Bermúdez-Humarán LG, Cortes-Perez NG, Loir YL, Gruss A, RodriguezPadilla AC, Saucedo-Cardenas O, et al. Fusion to a carrier protein and a synthetic propeptide enhances E7 HPV-16 production and secretion in Lactococcus lactis. Biotechnol Prog. 2003;19:1101-4.

23. Manjasetty BA, Chance MR. Crystal structure of Escherichia coli L-arabinose isomerase (ECAI), the putative target of biological tagatose production. J Mol Biol. 2006;360:297-309.

24. Rhimi M, Ilhammami R, Bajic G, Boudebbouze S, Maguin E, Haser R, et al. The acid tolerant L-arabinose isomerase from the food grade Lactobacillus sakei $23 \mathrm{~K}$ is an attractive D-tagatose producer. Bioresour Technol. 2010;101:9171-7.

25. Lu Y, Levin GV, Donner TW. Tagatose, a new antidiabetic and obesity control drug. Diabetes Obes Metabol. 2008;10:109-34.

26. Espinosa I, Fogelfeld L. Tagatose: from a sweetener to a new diabetic medication? Expert Opin Investig Drugs. 2010;19:285-94.

27. Sambrook J, Fritsh EF, Maniatis T. Molecular cloning: a laboratory manual. 2nd ed. Cold Spring Harbor: Cold Spring Harbor Laboratory Press; 1989.

28. Langella P, Le Loir P, Ehrlich DS, Gruss A. Efficient plasmid mobilization by pIP501 in Lactococcus lactis subsp. lactis. Appl Environ Microbiol. 1993;175:5806-13.

29. Rigoulay C, Poquet I, Madsen SM, Gruss A. Expression of the Staphylococcus aureus surface proteins HtrA1 and HtrA2 in Lactococcus lactis. FEMS Microbiol Lett. 2004;237:279-88.

30. Rhimi M, Bajic G, Ilhammami R, Boudebbouze S, Maguin E, Haser R, et al. The acid-tolerant L-arabinose isomerase from the mesophilic Shewanella sp. ANA-3 is highly active at low temperatures. Microb Cell Factor. 2011;10:96.

31. Laemmli UK. Cleavage of structural proteins during the assembly of the head of bacteriophage T4. Nature. 1970;227:680-5.

32. Dische Z, Borenfreund E. A new spectrophotometric method for the detection and determination of keto sugars and trioses. J Biol Chem. 1951;192:583-7.

33. Kuipers OP, de Ruyter PGGA, Kleerebezem M, de Vos WM. Quorum sensing-controlled gene expression in lactic acid bacteria. J Biotechnol. 1998;64:15-21.

34. Bermúdez-Humarán LG, Langella P, Miyoshi A, Gruss A, Guerra RT, Montes de Oca-Luna R, et al. Production of human papillomavirus type 16 E7 protein in Lactococcus lactis. Appl Environ Microbiol. 2002;68:917-22.

35. Cortes-Perez NG, Poquet I, Oliveira M, Gratadoux JJ, Madsen SM, Miyoshi A, et al. Construction and characterization of a Lactococcus lactis strain deficient in intracellular ClpP and extracellular HtrA proteases. Microbiology. 2006;152:2611-8. 